\title{
SOME REMARKS ON THE MODELLING OF THE THERMOMECHANICAL BEHAVIOUR OF SHAPE MEMORY ALLOYS
}

\section{LEXCELLENT and C. LICHT*}

Laboratoire de Mécanique Appliquée, Faculté des Sciences, Université de Franche-Comté, Route de Gray, La Bouloie, F-25030 Besançon cedex, France

*Laboratoire de Mécanique et de Génie Civil, Université des Sciences et des Techniques du Languedoc, case courrier 051, F-34095 Montpellier cedex 5, France

\begin{abstract}
Our aim is to examine from the continuum thermodynamic point of view, some models predicting the pseudoelastic behaviour of shape memory alloys. We investigate the choice of states variables, the structure of free energy and complementary laws. A special emphasis is made on generalized standard models. In the state of art, there is a lot of progress to do for modelling the behaviour of shape memory alloys.
\end{abstract}

\section{1. - INTRODUCTION.}

Our aim is to examine from the continuum thermodynamic point of view, some models predicting behaviour of shape memory alloys. In any case, we don't clain to be exhaustive, especially the attractive I. Muller [1] approach, based on statistic thermodynamic, is not described here. We present only some classical models. Moreover, we restrict our analysis to pure transformation plasticity. The main hypothesis is that the martensitic phase transformation is diffusionless in nature. We distinguish between two classes of models : generalized standard models and the other ones. For each one, we'11 investigate the choice of state variables, the structure of free energy and complementary laws. In the following $\varepsilon, \sigma, T$ are strains and stress tensors and temperature. The elementary language of convex analysis is suitable for formal items of thermodynamics [2], we use the following notions : if a state "ariable $\alpha$ is submitted to internal constraints, for instance, the volume fraction, it is convenient to consider that it is not only defined on a domain $C$ of $R^{n}$ but on the whole space, then free energy is the sum of the usual physical value ( $(O$ if $\alpha \notin C)$ and of the indicator function $I_{c}$ of $C . I_{c}(\alpha)=0$ if $\alpha \in C$, $I_{c}(\alpha)=+\infty$ if $\left.\alpha \notin C\right)$.

At last, the subdifferential $\partial f\left(x_{0}\right)$ of a convex function is a set of generalized gradients in $x_{0}: \partial f\left(x_{0}\right)=\left\{y \in R^{n}, f(x) \geq f\left(x_{0}\right)+y\left(x-x_{0}\right) \forall x \in R^{n}\right\}$. If $f$ is regular in $x_{0}, \partial f\left(x_{0}\right)$ is reduced to the gradient. $\partial \mathrm{I}_{c}\left(\mathrm{x}_{0}\right)$ is empty if $x_{0} \notin C$, is the outward normal cone if $x_{0}$ belongs to the boundary of $C$ and is reduced to (0) if $x_{0}$ belongs to the interior of $C$.

\section{2. -Generalized standard models.}

These models [3] are mainly characterized by the existence of a function D; called the pseudo-potential of dissipation, of state variable rates. D is convex minimal and equal to 0 in zero. They have the thermodynamic interest to satisfy automatically the Clausius Duhem inequality.

\section{$\underline{2} . \underline{1}$ Mode $\underline{1} \underline{\mathrm{M}} 1$}

A purely macroscopic model is presented in [4], using only directly observable state variables $(\varepsilon, \beta, T)$ where $\beta=\left(\beta_{1}, \beta_{2}\right)$ represents the volumic fraction of two variants of martensite. 
The expression of free energy of the mixture :

$$
\text { (1) } W(\varepsilon, \beta, T)=\left(\begin{array}{cc}
1-\sum_{i=1}^{2} & \beta_{i}
\end{array}\right) W^{A}(\varepsilon, T)+\sum_{i=1}^{2} \beta_{i} W^{M i}(\varepsilon, T)+I_{c}(\beta)
$$

means that it is the weighted average of free energies of the three phases. The contribution of the mixture appears only through $I_{c}$ with $C$ being the convex set $\left\{\beta_{i}\right.$, $\left.\beta_{i} \geq 0, \beta_{1}+\beta_{2} \leq 1\right) ;$ every interaction is neglected. In order to simplify and to restrict our purpose to the only problem of phase transformation, we consider that $D$ depends only on $\beta$. The behaviour laws remaining at constant temperatures are then :

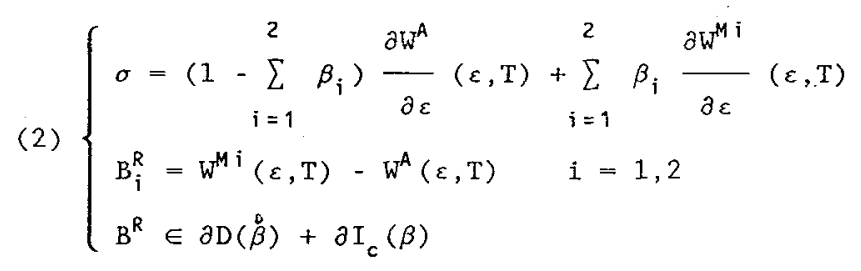

If $\mathrm{D}$ is regular in zero, the transformation $\mathrm{A} \rightarrow \mathrm{Mi}$ and $\mathrm{Mi} \rightarrow \mathrm{A}$ begins at the same level of strain ( the physical point of view. Thus, it is advisable to suppose that $D$ is non-smooth in zero, which would justify the vocable "transformation plasticity". Note also that a diffusion less transformation implies from (2) that $D$ is positively homogenous of degree 1 with respect to $\ddot{\beta}$. Another process [4] is to substitute the triangle $C$ by a curvilinear triangle included in $C$, which is an element of accounting the interactions. The fact that $B^{R}$ is independent of $\beta$, comes from the expression of the free energy of the mixture as the weighted average. Interaction is neglected, other models will try to consider them.

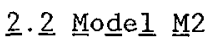

In [5] and [6] a model for monocristal with $n$ variants of martensite $\left(\beta=\left(\beta_{1}, \beta_{2} \ldots \beta_{n}\right)\right)$, is first presented. By means of reasonable physical assumptions, accounting cristallographic aspects, the structure of the free enthalpy is setted, so we can write it like this :

$$
\mathrm{w}^{*}(\sigma, \beta, \mathrm{T})=-\mathrm{w}_{\mathrm{ch}}(\mathrm{T})\left(\sum_{i=1}^{\mathrm{n}} \beta_{\mathrm{i}}\right)+\sigma\left(\sum_{\mathrm{i}} \mathrm{g} \mathrm{R}_{\mathrm{i}} \beta_{\mathrm{i}}\right)+\mathrm{w}_{\mathrm{e}}^{*}(\sigma)-\mathrm{w}_{\mathrm{I}}(\beta)-\mathrm{I}_{\mathrm{c}}(\beta)
$$

$w_{c h}, w^{*}$, $w_{i}$ are connected with chemical, elastic, interaction between variants ef fects, $g$ and $R$ with the kinematics of the transformation $C=\left\{\beta \in \mathbb{R}^{n}, 0 \leq \beta_{i}, \sum_{i} \beta_{i} \leq 1\right\}$. Hence the free energy is :

(3) $\mathrm{w}(\varepsilon, \beta, \mathrm{T})=\mathrm{w}_{\mathrm{e}}\left(\varepsilon-\sum_{\mathbf{i}} \mathrm{g} \mathrm{R}_{\mathbf{i}} \beta_{\mathrm{i}}\right)+\left(\sum_{i} \beta_{\mathrm{i}}\right) \mathrm{w}_{\mathrm{ch}}(\mathrm{T})+\mathrm{w}_{\mathrm{i}}(\beta)+\mathrm{I}_{\mathrm{c}}(\beta)$

From this expression, we can identify the inelastic (Phase Transformation) strain $\varepsilon^{P T}=\sum_{\mathbf{i}} \mathrm{g} \mathrm{R}_{\mathbf{i}} \beta_{\mathbf{i}}$ and observe that the energy is separated in an elastic part and a reversible stored one by internal state change where the interaction term $W_{1}$ will play an important role.

In the case of a non-dissipative behaviour, the isothermal laws are then :

$$
\sigma=\frac{\partial w_{e}}{\partial \varepsilon}\left(\varepsilon-\sum_{i} g R_{i} \beta_{i}\right)
$$


(5)

$$
\left\{\begin{array}{l}
B_{i}^{R}=g R_{i} \sigma-W_{c h}(T)-\frac{\partial W_{1}(\beta)}{\partial \beta_{i}} \quad i=1, \ldots, n \\
B^{R} \in \partial I_{c}(\beta)
\end{array}\right.
$$

A domain in the stress space in the interior of which there is no transformation is evidenced :

$$
g R_{\mathrm{i}} \sigma<\mathrm{w}_{\mathrm{ch}}(\mathrm{T})+\frac{\partial \mathrm{W}_{\mathrm{I}}(0)}{\partial \beta_{\mathrm{i}}} \quad \mathrm{i}=1, \ldots, \mathrm{n}
$$
gives :

For example, in the case of one variant and uniaxial test, (5)

$$
\left\{\begin{array}{l}
g R \sigma \leq w_{c h}(T)+w_{1}^{\prime}(0) \Rightarrow \beta=0 \\
w_{c h}(T)+w_{1}^{\prime}(0) \leq g R \sigma \leq w_{c h}(T)+w_{l}^{\prime}(1) \Rightarrow w_{I}^{\prime}(\beta)=g R \sigma-w_{c h}(T) \\
w_{c h}(T)+w_{I}^{\prime}(1) \leq g R \sigma \Rightarrow \beta=1
\end{array}\right.
$$

Henceworth, with a plausible assumption of strict convexity of energy, (7) determines in a unique way $\beta$ as a function of $(\sigma, T)$, and then $\varepsilon^{P T}=g R \beta$ and at last from (4), the total strain. We have to note that it is not necessary to write incremental equations to obtain the evolution of $\varepsilon^{\mathrm{PT}}$.

Such a non-dissipative behaviour cannot predict hysteresis during isothermal transformation, the same authors have proposed a solution by introducing two functions $W_{c h}^{A M}$ and $W_{c h}^{M A}$.

We think that the introduction of a pseudopotential of dissipation $D$ which is convex, minimal and equal to 0 in zero, depending only on $\dot{\beta}$ (once more for accounting only the effects of phase transformation) produces a generalized standard model which allows us to answer the previous criticisms. The isothermal behaviour laws become (4) and also :

$$
\mathrm{B}^{\mathrm{R}} \in \partial \mathrm{I}_{\mathrm{c}}(\beta)+\partial \mathrm{D}(\dot{\beta})
$$

In the case of regular dissipation in zero, the threshold transformation is still the one given by (6). Assuming once more that $W_{1}$ is strictly convex, the transformations $A \rightarrow M_{i}$ and $M_{i} \rightarrow A$ begin at different levels of strain and stresses which seems more realistic than the model M1. This is because the free energy depends of $\beta$ in a more complex way. The stresses $\sigma_{\mathrm{MS}}$ and $\sigma_{\mathrm{As}}$ depend only on $\mathrm{T}$, but $\sigma_{\mathrm{Mf}}$ and $\sigma_{\mathrm{Af}}$ depend also on the loading-unloading process.

In fact, under the assumption of diffusionless phase transformation, from (8), $D$ must be positive, homogeneous of degree 1 and thus non-smooth in zero. Then, the freedom given to $D$ by the generalized standard material theory, to depend also of state variables, allows us to predict in accordance with experimental observations, different kinetics and lines of transformation in $(\sigma, \mathrm{T})$ for direct transformation $\mathrm{A} \rightarrow$ $M$ and reverses $M \rightarrow A$. It is possible to identify $D$ and the components of $W$ by fitting W with experimental results.

In order to predict the polycristal behaviour a first investigation consists of homogenizing, by a self consistent method, the non-dissipative behaviour described by (4), (5) for monocristal. A second purely phenomenological approach [6] consists of introducing a criterium for initiation transformation $F(\sigma, T)=0$ and an evolution law for $\dot{\varepsilon}^{\mathrm{PT}}: \dot{\varepsilon}^{\mathrm{PT}}=\lambda \frac{\partial \mathrm{F}}{\partial \sigma}, \mathrm{F}(\sigma, \mathrm{T}) \geq 0 ; \lambda$ being identified through elementary mechanic tests. We think that in this model, it will be suitable to limit $\varepsilon^{\mathrm{Pr}}$ and to consider the reverse transformation.

It's at least formally possible to homogeneize the dissipative behaviour (4) and (8), but it is classical [8] that the structure of macroscopic constitutive laws 
can be more complex than (5) and (8). Nevertheless, in a first approximation a modelling of the macroscopic behaviour would be to keep a structure like (4) and (8).

State variables $\left(\varepsilon, \varepsilon^{P T}, T\right)$

Free energy $W\left(\varepsilon, \varepsilon^{P T}, T\right)=W_{e}\left(\varepsilon-\varepsilon^{P T}, T\right)+W_{a}\left(\varepsilon^{P T}, T\right)+I_{c}\left(\varepsilon^{P T}, T\right)$

Dissipation $D\left(\varepsilon^{P I}, \varepsilon^{P T}\right)$ positive, homogeneous of degre 1 in $\varepsilon^{P T}$

\section{$\underline{2} \cdot \underline{3} \underline{\operatorname{M}} \circ \underline{\mathrm{d}} \mathrm{e} \underline{\underline{\mathrm{M}} 3}$}

The structure of the free energy in the two previous models comes sical choices. The frame of generalized standard materials is flexible allow a very general structure as in the model [9]. $(\varepsilon, \beta, T)$ are the state from phy enough to variables and the potential of dissipation is quadratic in $\dot{\beta}$ and depends on the state variable considered in that case as parameters. We can therefore identify $W$ and $D$, a posteriori, by general physical assumptions and fit them with experimental results.

\section{3. - Other mode1s.}

For these models, complementary laws can't be expressed with a convex and positive dissipation potential. They are purely macroscopic models where ( $\left.\varepsilon, \varepsilon^{P T}, T\right)$ are the state variables. The pseudoelasticity or pure transformation plasticity can be treated as in the classical plasticity formalism but with two yield surfaces $f_{1}$ for $A \rightarrow M$ and $f_{2}$ for $M \rightarrow A$. We will classify the following models in function of the expression of the plastic multiplier $\lambda$.

\section{$\underline{3} . \underline{1}$ Model $\underline{M} 4 \_[\underline{1} 0]$}

The free energy is given by :

$$
W\left(\varepsilon, \varepsilon^{P T}, T\right)=W_{e}\left(\varepsilon-\varepsilon^{P T}, T\right)+W_{a}\left(\varepsilon^{P T}\right)+I_{c(T)}\left(\varepsilon^{P T}, T\right)
$$

The use of the indicator function is to bound $\varepsilon^{P T}$ inside a temperature dependent convex $C$. Its boundary corresponds to the end of the transformation $A \rightarrow M$. $f_{1}$ and $f_{2}$ are such that :

i) the yield surfaces $f_{1}(, T)=0$, divide the stress space in two connected parts

ii) $f_{1}(O, T)<0, f_{2}(\tau, T)=0 \Rightarrow f_{1}(\tau, T)<0$

iii) $f_{2}(O, T)<0$ (resp. $>0$ ) for $T$ low (resp. high)

Moreover, the second criterium responds only if $\left|\varepsilon^{P T}\right|$ will decrease. The normality rule and the consistency condition give the evolution of $\varepsilon^{p T}$. We have to note that the Clausius-Duhem inequality is not always satisfied, during unloading and reverse transformation.

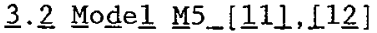

In [11], the choice of free energy is dual of the one of M1, the free enthalpy of the mixture is the weighted average of the constituent enthalpies. It's clear that the truth Iies between these two approaches...

The first criterium $(A \rightarrow M)$ is :

$\mathrm{f}_{1}=\bar{\sigma}-\dot{\bar{\sigma}}_{\mathrm{Ms}}$ with $\left(\bar{\sigma}=\left(\begin{array}{c}3 \\ -\operatorname{dev} \sigma: \operatorname{dev} \sigma \\ 2\end{array}\right)^{1 / 2}\right.$,

The second criterium for $M \rightarrow A$ is :

$f_{2}=\bar{\sigma}_{A S}-\bar{\sigma}$ in [12] and $f_{2}=\operatorname{dev} \sigma: \varepsilon^{P T}-\bar{\sigma}_{A S} \vec{\varepsilon}^{P T}$ in [11]. 
The direction of $\varepsilon^{p T}$ is dev $\sigma$ during the direct transformation. For the reverse one, $\varepsilon^{P T}$ has the direction of dev $\sigma$ in [12] and of (dev $\sigma-\alpha \varepsilon^{P T}$ ) (with $\left.\alpha \frac{3}{2}\left|\varepsilon^{p \mathrm{~T}}\right|=\frac{\bar{\sigma}_{\mathrm{AS}}+\bar{\sigma}_{M S}}{2}\right]$ in [11].

At last, the martensitic proportion $\beta$ is the ratio of the actual $\bar{\varepsilon}^{\mathrm{PT}}$ to total transformation strain when phase transformation $A+M$ which occured perfectly during the tensile test. This permits us to connect the modulus of $\varepsilon^{P r}$ to classical expression of phase transformation kinetics.

\section{CONCLUSION}

Even if a fairly good agreement between experimental [6] [12] and predicted results is obtained, modelling of phase transformation effects are not yet completely understood.

For instance, volume proportion of martensite seems to be a measurable variable which is operative for macroscopic modelling but not sufficient because of its scalar character.

An internal tensoriel variable linked to pure transformation strain would be more appropriate. The real dissipative phenomena (heat dissipation by interfaces friction) should receive a sharp physical analysis in order to be better integrated in the complementary law expressions. Otherwise, the time effects shown by creep and relaxation tests [12] don't seem negligible. Accounting them, will deal with "pure transformation viscoplasticy".

At last, we have to extend these models by integrating training processes which are essential for technological use of these shape memory alloys.

\section{References}

[1] MULLER I., Arch. Rat. Mec, Ana1. 70, (1979), 61

[2] GERMAIN P., "Cours de Mécanique des Milieux Continus" Masson - Paris, (1973)

[3] HALPHEN B., N'GUYEN Q.S., J. de Meca. 14, (1975)

[4] FREMOND M., CRAS 304, II, 7, (1987), 239

[5] PATOOR E., BERVEILLER M., "Les alliages à mémoire de forme", Hermès, (1990)

[6] PATOOR E., EBERHADT A., BERVEILleR M., Acta. Met. 38, (1987), 2779

[7] PATOOR E., EBERHADT A., BERVEILLER M., Proceedings of Mecamat, Besançon, (1988)

[8] SUQUET P., Thèse, Paris VI, (1982)

[9] TANAKA $K_{\text {. }}$, KOBAYASHI S., SATO Y., Int. J. of Plast. 3, (1986), 59

[10] BERTRAM A., Nucl. Eng and Design 74, (1982), 17

[11] WANG Z.G., Proceedings of ICRS 2, Nancy, (1988), 529

[12] VACHER P., Thèse, Besançon, (1991) 